\title{
Wavepacket eduction in turbulent jets based on eigenmode decomposition of PIV data
}

\author{
Daniel Rodríguez ${ }^{1,2 *}$ André V. G. Cavalieri ${ }^{3 \dagger}$ Tim Colonius ${ }^{1 \ddagger}$ and Peter Jordan ${ }^{4 \S}$, \\ ${ }^{1}$ California Institute of Technology, Pasadena, $C A$ \\ ${ }^{2}$ School of Aeronautics, Universidad Politécnica de Madrid, Spain \\ ${ }^{3}$ Instituto Tecnológico de Aeronáutica, São José dos Campos, Brazil \\ ${ }^{4}$ CNRS - Université de Poitiers - ENSMA, France
}

The dynamics of large scale structures in unforced turbulent jets at subsonic speeds have been related to the generation of the peak noise radiated the aft direction. The utility of instability wavepackets computed by linear stability theory or parabolised stability equations (PSE) have been demonstrated for the modeling of the near-field pressure fluctuations associated with the coherent structures. In this paper, we investigate whether the velocity field corresponding to the wavepackets also represents adequately that of the coherent structures. Previous research showed remarkable agreement in the velocity field up to the end of the potential core, but the agreement is lost gradually downstream.

Locally-parallel linear stability theory (LST) of jet velocity profiles is revisited to further study the evolution of the wavepackets and the manner in which PSE models them. An adjoint-based eigenmode decomposition technique is used to project cross-sectional velocity profiles measured using time-resolved particle image velocimetry (PIV) on the Kelvin-Helmholtz eigenmode responsible for the wavepacket amplification. The instability wave thus extracted is then compared, both in amplification and shape, to the PSE wavepacket and to the dominant coherent structures obtained from the proper orthogonal decomposition of the PIV measurements. The comparisons between PSE models and POD-filtered fluctuations define three spatial regions along the streamwise direction that are explained in terms of changes in the LST eigenspectrum.

\section{Introduction}

The noise generated by turbulent jets and its subsequent radiation to the far field is a technological problem of great importance that has received continuous attention for decades. Investigation of the associated flow physics numerically, using direct numerical simulations or large eddy simulations at Reynolds and Mach numbers of practical application remains challenging and computationally expensive even with state-of-the-art algorithms ${ }^{1}$ and supercomputers. An intermediate approach is born from the observation ${ }^{2,3}$ that the highly-directional peak noise radiated in the aft direction of turbulent jets is associated with the dynamics of the large-scale coherent structures. Relatively coarse descriptions of the large-scale structures combined with acoustic projection methods are then suggested as a promising method for the prediction of the noise in the aft direct.

Statistical descriptions of the coherent structures observed in jets are reminiscent of instability waves, suggesting their modeling as instability wavepackets of the mean turbulent flow. ${ }^{4,5}$ Most of the studies comparing wavepackets in turbulent jets with stability theory considered forced jets, for which the introduction of controllable phase-locked disturbances enables a direct comparison between experiments and theoretical

\footnotetext{
* Marie Curie COFUND fellow, Present address: Escola de Engenheria de São Carlos, Universidade de São Paulo, Brazil and School of Aeronautics, Universidad Politécnica de Madrid, Spain. AIAA Member. dani@torroja.dmt.upm.es

†Assistant Professor, Divisão de Engenharia Aeronáutica, Praça Mal. Eduardo Gomes, 50, 12228-900 São José dos Campos, SP, Brazil

${ }^{\ddagger}$ Professor, Department of Mechanical Engineering, California Institute of Technology, USA, AIAA Associate Fellow.

$\S$ Research Scientist, Institut Pprime, 43 rue de l'Aérodrome, 86036 Poitiers, France.
} 
models; the predictions of linear stability theory ${ }^{6}$ for the statistical description of the wavepackets were found to be in good agreement with the measured near-field fluctuations. ${ }^{4,7}$ In the case of natural or unforced jets, the lack of a phase reference precluded the obtention of satisfactory quantitative comparisons between theoretical wavepackets and the experimental data until relatively recently. Suzuki and Colonius ${ }^{10}$ considered a series of measurements performed at the NASA Glenn SHJAR facility, in which a microphone phased-array was carefully placed just outside of the turbulent mixing-layer, in a region where hydrodynamic fluctuations are expected to behave linearly and the relative importance of the acoustic fluctuations to be minimal. This experimental technique, almost identical to the one used by Tinney and Jordan ${ }^{11}$ and more recently by Breakey et al. ${ }^{12}$ for the same jet configurations considered in this work, was instrumental in the successful eduction of the near-field pressure fluctuations corresponding to the coherent structures, leading to remarkable comparisons with the theoretical wavepackets.

A step beyond the use of parallel-flow linear stability theory in the modeling of the wavepackets is achieved with the introduction of the Parabolised Stability Equations (PSE). ${ }^{13,14}$ In our previous works,${ }^{15-18}$ we employed PSE to take into account the mild variation of the jet mean flow along the axial direction. The use of the experimental turbulent mean flow as base flow in the PSE computations partially accounted for the nonlinear interactions present in the flow, resulting in good comparison with near-field pressure measurements. Recently, ${ }^{15,18}$ we investigated the presence of wavepackets in the velocity field of turbulent jets. When considering natural jets, the signature of the azimuthally-coherent wavepackets is clearer in the near pressure field, whose structure is considerably simpler than that of the turbulent velocity field: the energy of the hydrodynamic pressure field is concentrated in the few low-order azimuthal modes, in clear contrast to the velocity field. ${ }^{18,19}$ On the other hand, the signature of the wavepackets in the velocity field of forced turbulent and transitional jets is relatively clear. ${ }^{2,20}$ Its eduction in natural jets is more difficult due to the lack of a phase reference, but also to the smaller energy content of the coherent structures compared to the total velocity fluctuations. Gudmundsson and Colonius ${ }^{15}$ compared the velocity field of unforced jets measured using particle image velocimetry (PIV) in cross sections with the results of linear PSE showing an encouraging agreement.

Cavalieri et al. ${ }^{18}$ pursued further this issue by combining hot-wire anemometry and stereoscopic timeresolved PIV in cross-stream planes for measuring the velocity field and a microphone ring array at a polar angle of $\theta=20^{\circ}$ for the sound radiation. Correlations between the velocity and the acoustic pressure signals, previously decomposed into azimuthal Fourier modes, around $10 \%$ were found, being significantly higher than those reported previously for two-point flow-acoustic correlations. This result suggests that, despite the broadband character of turbulent structures in the velocity field in which higher azimuthal modes dominate, velocity fluctuations exist at the lowest azimuthal modes that are intimately related to the noise emitted to the far-field. In the same work, the axisymmetric and first helical modes of the velocity field were compared with PSE wavepackets. For all but the lowest frequencies considered, close agreement was found in the spatial amplification, up to the end of the potential core. The radial fluctuation profiles delivered by PSE also agreed with the experimental results over the same region. However, the agreement is gradually lost downstream of the potential core.

The present work revisits the velocity field associated with the large-scale structures present in turbulent jet flows. Results from the locally-parallel linear stability theory (LST) are used in order to further study the evolution of the wavepackets and the manner in which PSE models them. While the true physics in the turbulent jet flow are evidently much more involved than simple linear dynamics, the interpretation of the wavepacket evolution in terms of a set of well-understood eigenmodes serves us to shed light on some fundamental aspects. In addition, an eigenmode decomposition algorithm of the PIV data is presented. Analogous eigenmode decompositions of experimental and simulation data have been performed in the past in the study of laminar and transitional flows, ${ }^{21-24}$ and recently for supersonic turbulent jets. ${ }^{25}$ Attention is focused on the evolution of the Kelvin-Helmholtz $(\mathrm{K}-\mathrm{H})$ eigenmode and the projection of experimental fluctuations on it. In subsonic jets, the inflectional K-H instability is the only possible mechanism for linear amplification of the large-scale fluctuations, and governs the wavepacket evolution. Comparisons of the fluctuations extracted as the projection of the K-H eigenmode with PSE solutions and experimental velocity measurements are done, revealing a deep connection between the downstream evolution of the properties of the LST eigenspectrum and the measured velocity fields. 
a)

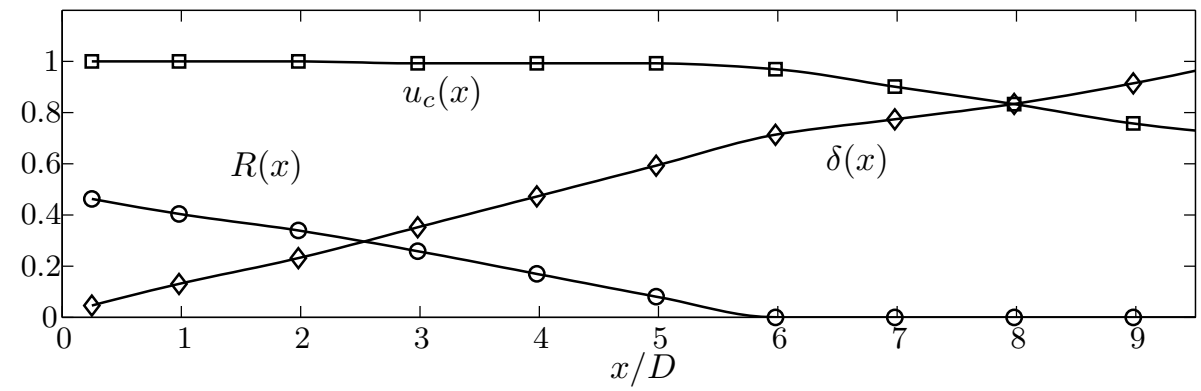

b)

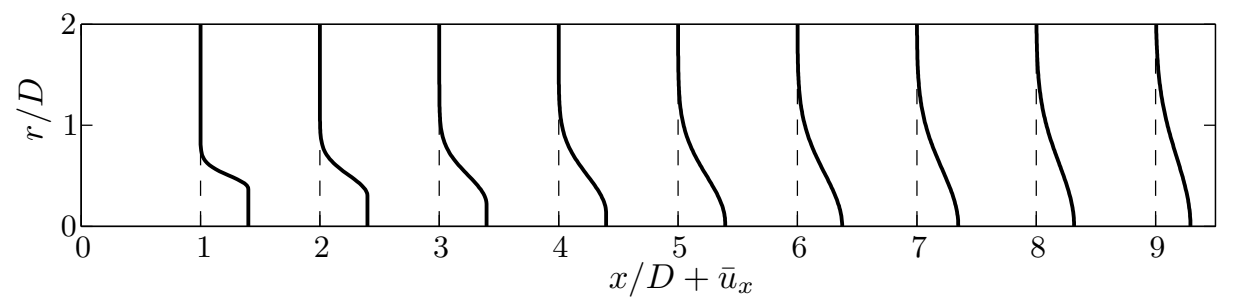

Figure 1. Profile parameters determined from experiment (a). Mean flow profiles for axial velocity component $\overline{u_{x}}(\mathbf{b})$.

\section{Flow configuration and experimental setup}

An isothermal subsonic round jet with exhaust Mach number equal to 0.4 and Reynolds number based on the exhaust velocity and nozzle diameter equal to $R e=U_{j} D / \nu=4.2 \times 10^{5}$ is considered here. This flow configuration was studied in a series of experiments performed in the 'Bruit et Vent' anechoic facility at the Centre d'Etudes Aérodynamiques et Thermiques (CEAT), Institut Pprime, Poitiers, France. These experiments were specifically design in order to study the existence of wavepackets in the unforced turbulent jet flow, and their relevance to the highly directional noise radiation at aft angles. Different aspects have already been addressed and reported ${ }^{18,26}$ and in ongoing efforts. ${ }^{12}$ A detailed presentation of the experimental facility, measurement equipment and data processing can be found in the given references; only the information most relevant to the present work is repeated in what follows.

A convergent section was located upstream of the jet axis with an area contraction of 31, followed by a straight circular section of length $150 \mathrm{~mm}$ and diameter $D$ equal to $0.05 \mathrm{~m}$. A carborundum trip placed 2.7D upstream of the nozzle exit was used to enforced transition of the inner boundary layer before the lip, ensuring that the annular jet mixing-layer is initially turbulent.

Hot-wire measurements were made to determine the mean axial velocity field. A sufficient measurement time is used in order to eliminate the influence of transients. However, to avoid numerical issues in the subsequent numerical computations stemming from residual non-smoothness in the measured field, the mean flow is fitted with a Gaussian profile ${ }^{3,15,27 a}$ :

$$
\bar{u}_{x} / U_{j}= \begin{cases}1 & \text { if } r<R(x) \\ u_{c}(x) \exp \left[-(r-R(x))^{2} / \delta^{2}(x)\right] & \text { otherwise }\end{cases}
$$

The parameters $u_{c}, R$ and $\delta$ are determined by a least-squares fitting of the experimental data. A zero mean pressure gradient is assumed, and the Crocco-Busemann relation is used to determine the mean temperature and density fields. The radial velocity field is then obtained from the continuity equation. Figure 1 shows the main features of the jet mean velocity field.

Velocity measurements along the jet centerline were obtained using a traversing single hot wire. Stereoscopic, time-resolved particle image velocimetry (PIV) measurements were performed at different cross planes. The sampling frequency was $5 \mathrm{kHz}$, corresponding to $S t=f D / U_{j}=1.82$ for the present jet; this

${ }^{\text {a }}$ The present expression differs from that in References 3 and 27 in a factor $\ln 2$ that is included in $\delta$. 
frequency was the maximum possible value with available equipment. Details on the experimental setup, PIV processing and the evaluation of possible errors due to aliasing can be found elsewhere. ${ }^{18}$ In the present study we focus the analysis of the jet measurements on the Strouhal number range of $0.1 \leq S t \leq 0.9$ to diminish significant errors due to aliasing, but some noticeable effects can still be observed at the higher frequencies. The fluctuation velocity profiles used in the eigenmode projection are obtained from the shorttime discrete Fourier transform of the PIV data. The time segment length used in the Fourier transform corresponds to a frequency bin $\Delta S t=0.025$, resulting in 523 segments with $50 \%$ overlap. During the first phase of analysis of the results presented herein, it was found that 100 segments suffice to attain very good convergence of the statistical velocity profiles.

\section{Theoretical background}

This section briefly describes the approach followed for the modeling of the large-scale and low-frequency fluctuations in natural turbulent jets. A broader discussion on the approach and on the numerical implementation can be found elsewhere. ${ }^{15,25}$ The turbulent flow field is decomposed into a time-averaged (or mean) flow and temporal fluctuations, $\mathbf{q}(\mathbf{x}, t)=\overline{\mathbf{q}}(\mathbf{x})+\mathbf{q}^{\prime}(\mathbf{x}, t)$. A cylindrical coordinate system is used where $\mathbf{x}=(x, r, \theta)$ are the axial, radial and azimuthal coordinates respectively. The vector of fluid variables is denoted by $\mathbf{q}=\left[u_{x}, u_{r}, u_{\theta}, p, \zeta\right]$, where $u_{x}, u_{r}$ and $u_{\theta}$ are the axial, radial and azimuthal velocity components, $p$ is pressure and $\zeta$ is the specific volume, inverse of the density. Fourier modes are introduced for frequency $\omega=2 \pi M_{j} S t$ and wavenumber $m$ following

$$
\mathbf{q}^{\prime}(\mathbf{x}, t)=\sum_{\omega} \sum_{m} \breve{\mathbf{q}}_{\omega m}(x, r) \mathrm{e}^{\mathrm{i} m \theta} \mathrm{e}^{-\mathrm{i} \omega t} .
$$

\section{A. Parabolised stability equations}

Owing to the slowly divergent nature of the mean flow along the axial direction, parabolised stability equations (PSE) have been used in the past ${ }^{15,18,28-31}$ as a model for the spatial evolution of the large-scale wave packets. The fluctuations $\breve{\mathbf{q}}_{\omega m}$ are decomposed into a slowly-varying shape function, evolving in the same scale as the mean flow, and a rapidly varying wave-like part:

$$
\breve{\mathbf{q}}_{\omega m}(x, r)=A_{\omega m}(x) \tilde{\mathbf{q}}_{\omega m}(x, r)=A_{\omega m}\left(x_{0}\right) \exp \left(\mathrm{i} \int_{x} \alpha_{\omega m}(\xi) d \xi\right) \tilde{\mathbf{q}}_{\omega m}(x, r) .
$$

The axial wavenumber $\alpha_{\omega m}=\alpha_{r}+\mathrm{i} \alpha_{i}$ is a complex quantity for which a slow variation is also assumed. Introducing this decomposition into the compressible Navier-Stokes, continuity and energy equations and subtracting the terms corresponding to the mean flow we arrive at the system of equations

$$
\left(\mathbf{A}+\mathbf{B} \frac{d \alpha}{d x}+\mathbf{C} \frac{\partial}{\partial x}+\mathbf{D} \frac{\partial}{\partial r}+\mathbf{E} \frac{\partial^{2}}{\partial r^{2}}+\mathbf{F} \frac{\partial^{2}}{\partial x \partial r}\right) \tilde{\mathbf{q}}_{\omega m}(x, r)=R_{\omega m} .
$$

The linear operators $\mathbf{A}$ to $\mathbf{E}$ depend on the mean flow quantities, Reynolds number, Mach number, frequency $\omega$ and azimuthal wavenumber $m$ and axial wavenumber $\alpha$. The system of equations (4) is a linear spatial operator for each mode $(\omega, m)$ with the forcing term $R_{\omega m}$ accounting for mode-dependent Reynolds stresses. For unforced turbulent jets, the small relative amplitude of the individual modes suggests that nonlinear interactions between the lower modes can be neglected, as most nonlinear effects are implicit in the mean flow, and then $R_{\omega m} \approx 0 .{ }^{32}$

After spatial discretization of the radial direction and imposition of adequate boundary conditions, the system (4) takes the form

$$
\mathbf{L} \frac{\partial \tilde{\mathbf{q}}_{\omega m}}{\partial x}=\mathbf{R} \tilde{\mathbf{q}}_{\omega m}
$$

where $\mathbf{L}=\mathbf{C}+\mathbf{F} \mathcal{D}_{r}$ and $\mathbf{R}=-\left(\mathbf{A}+\mathbf{B} d \alpha / d x+\mathbf{D} \mathcal{D}_{r}+\mathbf{E} \mathcal{D}_{r r}\right)$ have been introduced. The matrix operators $\mathcal{D}_{r}$ and $\mathcal{D}_{r r}$ stand for the discrete versions of the first and second order spatial differentiation along the radial direction, respectively. The system of equations is discretised using fourth-order central finite differences in the radial direction, closing the domain with the characteristic boundary conditions of Thompson. ${ }^{33}$ The boundary conditions at the centerline are derived following Mohseni \& Colonius. ${ }^{34}$ 
The decomposition of (3) is ambiguous in that the spatial growth can be absorbed into the shape function $\tilde{\mathbf{q}}_{\omega m}$ or the complex amplitude $A_{\omega m}$. Following, ${ }^{14}$ the normalization condition

$$
\int_{0}^{\infty} \tilde{\mathbf{u}}_{\omega m}^{*} \frac{\partial \tilde{\mathbf{u}}_{\omega m}}{\partial x} r d r=0
$$

where $\tilde{\mathbf{u}}_{\omega m}$ refers to the three velocity components and $*$ denotes complex conjugation, is imposed individually to every $(\omega m)$ mode, removing the exponential dependence from $\tilde{\mathbf{q}}_{\omega m}$.

Expression (5) is an initial value problem to be integrated along the axial direction requiring of adequate inlet conditions. Specifically, the perturbation shape $\tilde{\mathbf{q}}_{\omega m}$ and wavenumber $\tilde{\alpha}_{\omega m}$ must be given at the inlet location $x_{0}$. The amplitude factor $A_{\omega m}$ can be determined a posteriori, as linear PSE results are independent of amplitude. Information provided by locally-parallel linear stability theory is usually employed in the determination of the inlet conditions.

\section{B. Local stability eigenvalue problem}

The local stability eigenvalue problem (EVP) is derived here from the PSE approximation, by assuming $d \alpha_{\omega, m} / d x \approx 0$ and $\partial \breve{\mathbf{q}}_{\omega, m} / \partial x \approx \mathrm{i} \alpha_{\omega, m} \breve{\mathbf{q}}_{\omega, m}$. These assumptions are the usual ones in the derivation of local stability problems of the Orr-Sommerfeld kind, but two differences exist. First, the axial derivatives of the mean flow that are retained in PSE are also retained here. As shown by Gudmundsson and Colonius, ${ }^{15}$ this approximation has a very limited effect. Second, the second axial derivatives appearing in the viscous term are neglected here as is done in PSE. These terms yield to $\alpha^{2}$ terms in the usual spatial stability problem, requiring of an special treatment to recast the equations as a linear EVP. Neglecting these terms alters the results from LST by eliminating two branches of upstream propagating vortical and entropic waves in the eigenspectrum ${ }^{35}$ leaving the other families practically unaltered. From the approximations above, the following matrix EVP results:

$$
\mathrm{i} \alpha \mathbf{L} \breve{\mathbf{q}}=\mathbf{R} \breve{\mathbf{q}},
$$

where the subscripts ( $\omega m$ ) have dropped for simplicity. Operators $\mathbf{L}$ and $\mathbf{R}$ are those in (5), but particularised for $\alpha=0$. The eigenvalue problem (7) describes the spatial growth or decay of disturbance waves with a fixed real frequency $\omega$ and azimuthal wavenumber $m$.

The solution of (7) delivers a complete eigenspectrum of solutions. For subsonic jets, only the discrete eigenmode corresponding to the Kelvin-Helmholtz mechanism may exhibit spatial growth. The present numerical solution of the problem leads to a discretization of the continuous branches and only discrete eigenmodes $\left(\alpha_{n}, \hat{\mathbf{q}}_{n}\right)$ are recovered. The properties of the LST eigenspectrum will be discussed in section IV.

\section{Bi-orthogonal decomposition and adjoint LST problem}

As was demonstrated for a flat-plate boundary layer, the eigenspectrum from LST is a complete set and an arbitrary perturbation profile can be decomposed exactly as a linear superposition of the eigenfunctions $\breve{\mathbf{q}}_{0}(r)=\sum_{n} a_{n} \hat{\mathbf{q}}_{n}(r) .{ }^{36-38}$ Due to the nonnormality of the linearised Navier-Stokes equations, the eigenfunctions do not conform an orthogonal system, and the solutions of the adjoint problem are required in order to obtain a bi-orthogonality relation. The adjoint eigenfunction corresponding to the discrete eigenmode $n$ is denoted by $\hat{\mathbf{q}}_{n}^{+}$. By construction of the adjoint EVP, between the direct and adjoint eigenfunctions holds the orthogonality relation

$$
\left(\hat{\alpha}_{n}-\hat{\alpha}_{l}\right)\left(\hat{\mathbf{q}}_{n}^{+}\right)^{*} \mathbf{L} \hat{\mathbf{q}}_{l}=0,
$$

that can be used to obtain the weighting coefficients $a_{n}$ in the projection of an arbitrary perturbation profile $\breve{\mathbf{q}}_{0}(r)$ :

$$
a_{n}=\left(\left(\hat{\mathbf{q}}_{n}^{+}\right)^{H} \mathbf{L} \breve{\mathbf{q}}_{0}\right) /\left(\left(\hat{\mathbf{q}}_{n}^{+}\right)^{*} \mathbf{L} \hat{\mathbf{q}}_{n}\right) .
$$

The maxima in the adjoint eigenfunctions correspond the spatial regions where each eigenmode is more receptive and serve as a filter of the relative contribution of the individual eigenmodes on the arbitrary perturbation profile. ${ }^{23,39-41}$ In particular, this decomposition represents a theoretically-founded method for the determination of the amplitude associated with the Kelvin-Helmholtz eigenmode at the different 
a) $x=0.25 D$

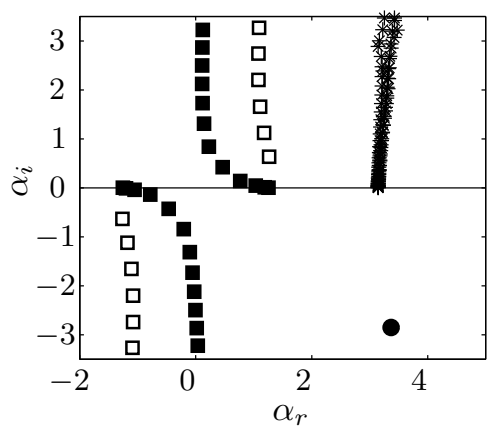

b) $x=3 D$

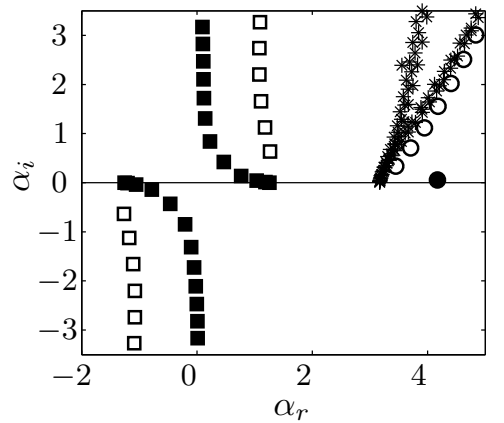

c) $x=6 D$

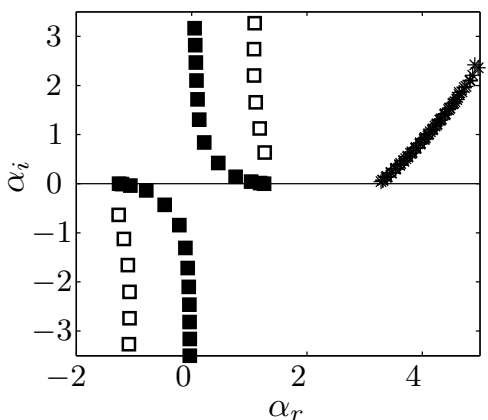

Figure 2. Spatial LST eigenspectra corresponding to axial locations $x=0.25 D, 3 D$ and $6 D$, representative of regions I, II and III respectively, for the Fourier mode $S t=0.5, m=0$. Filled circles: K-H eigenmode; open circles: shear-layer eigenmodes; Asterisks: Core eigenmodes; squares: acoustic eigenmodes.

measured cross-sections. The bi-orthogonal projection of empirical data (from experiments or high-fidelity numerical simulations) at a single cross-section in the vicinity of the nozzle exit has recently been shown to be a valid method for the amplitude calibration of linear wavepacket models. ${ }^{25,42}$

\section{Properties of the eigenspectrum}

The solution of the locally-parallel linear stability EVP (7) delivers a complete eigenspectrum of waves. Several studies in the past addressed the eigenspectrum of jet-related velocity profiles, either in the compressible inviscid, ${ }^{43,44}$ viscous incompressible ${ }^{45}$ and viscous compressible limits. ${ }^{46}$ Most of these studies focused on the instability of top-hat shaped velocity profiles, representative of the first diameters in the axial direction. However, due to the spread of the mean mixing-layer and the closure of the potential core, the properties of the eigenspectrum change significantly along the axial direction and deserve some discussion.

Three distinct spatial regions exist in the development of the mean turbulent jet, ${ }^{45,47}$ that can be described quantitatively using the analytic profile (1). Immediately downstream of the nozzle lip, a thin (but turbulent) mixing-layer exists surrounding a well-defined potential core. Top-hat velocity profiles are excellent approximations of Region I $(\delta \ll D, R \approx 0.5 D)$. In this region, theoretical considerations based on self-similarity of the velocity profile show that $u_{c}$ is constant and $\delta$ grows linearly with $x$. Region III starts downstream of the potential core, and corresponds to a fully developed turbulent jet in which the mixing-layer width is of the same order of magnitude of the jet diameter $(\delta \sim D, R \approx 0)$. A self-similarity solution also exists for this region, dictating that $u_{c}$ must decay as $\sim 1 / x$ and $\delta$ must grow linearly with $x$. Between Regions I and III lies a transition region, (referred here as Region II), in which a narrow potential core still exists, but $\delta$ is already of the same of magnitude as the nozzle diameter. No similarity solution is possible for this region. The three different regions are readily identified in figure 1(a).

The qualitative differences existing in the mean velocity profiles corresponding to the different regions yield important changes in the LST eigenspectrum, that also impact the axial evolution of the PSE solutions. Figure 2 shows representative eigenspectra of the three spatial regions. Some eigenfunctions corresponding to the different eigenmode branches are shown in figure 3 .

\section{A. Region I: annular mixing-layer, $\delta \ll D, R \approx 0.5 D$}

The top-hat velocity profile presents three distinct spatial zones along the radial direction: a well-defined potential core, a thin annular mixing-layer and the outer flow, i.e. the uniform unbounded stream surrounding the jet. An eigenspectrum representative of this region is shown in figure 2(a).

The outer flow supports a continuous spectrum of acoustic waves. ${ }^{3}$ The acoustic waves are of inviscid nature and correspond to solutions of the Helmholtz equation, describing a bounded continuum of waves propagating along the axial direction with supersonic velocity and an infinite continuum of evanescent waves ${ }^{44}$ (filled squares in figure 2). Figures 3(h) and 3(i)depict typical acoustic eigenfunctions. Two additional branches present in the computed eigenspectra (open squares in figure 2) are classified as acoustic waves too. Helmholtz equation admits solutions for which the radial and axial velocities vanish, and the only 
non-zero component is the azimuthal velocity. These waves are evanescent and propagate with speed of sound relative to the mean flow. In the discretised version, finite resolution distorts these branches towards higher phase speeds. In any case, the practical relevance of these waves is negligible in the present case, as non-axisymmetric fluctuations are not imposed to the jet.

The potential core can be regarded as a bounded uniform flow, supporting infinite discrete equivalents of the continuous spectra. Of particular significance in the present work are the branches of vorticity and entropy waves, of viscous origin. These waves are stable and propagate either upstream or downstream with phase velocity equal to the mean velocity in the potential core, i.e. to the jet exit velocity. The approximation of neglecting the second-order axial derivative terms in the EVP neglects the upstream propagating branches ${ }^{35}$ and consequently they are not recovered in the present eigenspectrum. The eigenmodes corresponding to these branches are denoted by asterisks in figure 2, and two eigenfunctions are shown in figures 3(f) and $3(\mathrm{~g})$.

Finally, the inflectional mixing-layer supports the discrete eigenmode corresponding to the K-H instability mechanism (denoted a filled circle in the figures). In subsonic jets, $\mathrm{K}-\mathrm{H}$ is the only potentially unstable eigenmode, as opposed to supersonic jets that can support additional kinds of modal instability. ${ }^{44}$ The K-H eigenmode is always unstable for the frequency range of interest over Region I.

\section{B. Region II: transition, $\delta \sim D$, small $R$}

Region II is characterised by a relatively thick mixing-layer, comparable to the size of the potential core and the jet diameter, leading to qualitative changes in the inflectional instability waves. An important parameter in the analysis of mixing-layer instability ${ }^{48}$ relates the frequency $\omega$ to the spatial scale of the layer (here $\delta$ ); the EVP (7) can then be rewritten for the rescaled frequency $\omega \delta$. In Region I, the small value of $\delta$ translates into $\omega \delta \ll 1$ for the range of frequencies of interest, and the single discrete eigenmode corresponding to Kelvin-Helmholtz theory appears. In region II, $\omega \delta$ takes values of magnitude $O(1)$ (between 0.1 and 1.7), depending on the frequency and axial location. A representative eigenspectrum of this region is shown in figure 2(b), corresponding to $x=3 D$ and $S t=0.5(\omega \delta \approx 0.44)$.

When $\omega \delta \sim 1$, the mixing-layer is relatively thick and permits the existence of more than a single discrete eigenmode associated to it: a new branch of discrete mixing-layer eigenmodes appear in the eigenspectrum (denoted by the open circles close to the branch of core eigenmodes). When the rescaled frequency is small, one eigenmode - the Kelvin-Helmholtz - is unstable, and clearly distinguishable from the others. As $\omega \delta$ increases, the K-H eigenmode first becomes stable and then eventually becomes part of the branch. The eigenfunctions corresponding to this branch (figures 3(c-e)) show localised oscillations in the mixing-layer; as for the other branches, the eigenmodes can be enumerated following the number of peaks or nodes that the eigenfunction presents within this region.

The description of viscous branches of vortical and entropic waves within the potential core also changes with increasing axial coordinate. As the velocity distribution diverges from the uniform flow, the branch is distorted from the nearly vertical line in a tilted line towards higher real wavenumbers, or equivalently, lower phase speeds. The outer-field acoustic branches remain nearly unaltered along the axial direction, as the mixing region spread does not modify substantially the outer region supporting them.

\section{Region III: developed jet, $\delta \sim D, R=0$}

Region III starts at the end of the potential core $(R \approx 6 D)$, and extends downstream. The velocity profile presents two zones with a smooth transition: the outer field and a thick mixing-layer $(\omega \delta>1)$ extending from the jet axis. The viscous branches associated with the potential core and the mixing-layer eigenmodes coalesce into a single group of eigenmodes, with amplitude functions as those shown by Figure 3 in Morris ${ }^{45}$ and figures $3(\mathrm{f})$ and $3(\mathrm{~g})$; these eigenfunctions are qualitatively identical to those of the core vorticity branch in regions I and II. Again, the changes in the mean profile in this region do not alter significantly the eigenmodes in the acoustic branches. Figure 2(c) shows the eigenspectrum corresponding to $x=6 D$ and $S t=0.5$.

\section{Bi-orthogonal projection of the PIV data}

The bi-orthogonal projection discussed in section III.C can be used in order to decompose the experimental data into separate contributions of the different eigenmodes from linear stability theory. In this section, 
a) Mean profile

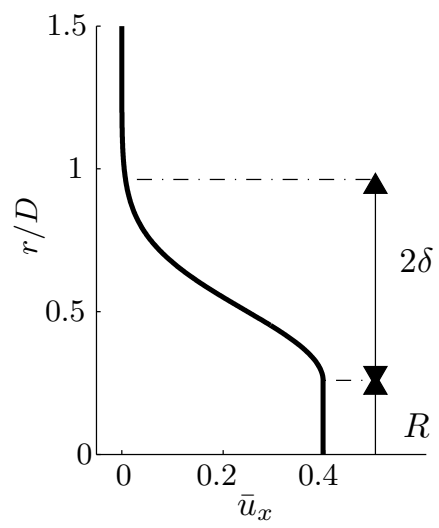

d) Shear-layer mode 2

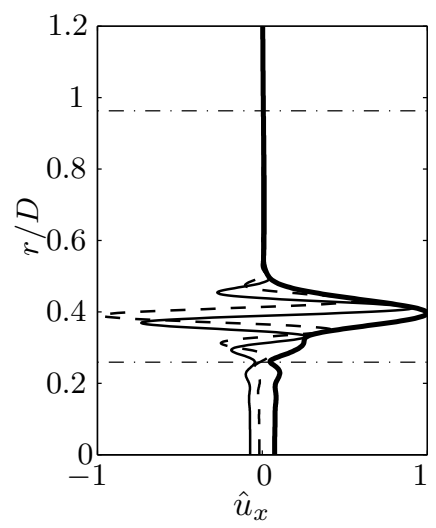

g) Core vorticity mode 2

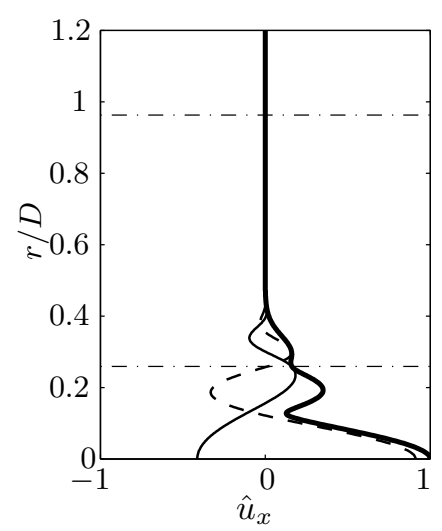

b) K-H mode

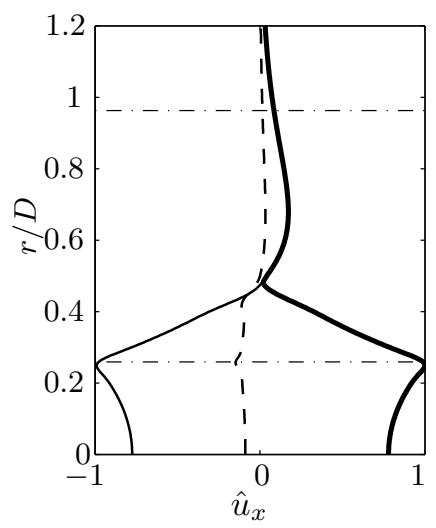

e) Shear-layer mode 3

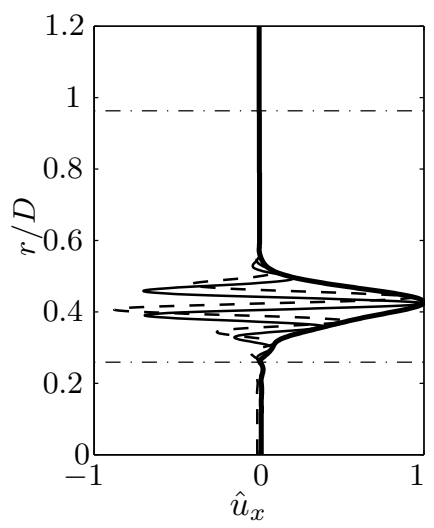

h) Acoustic mode 2

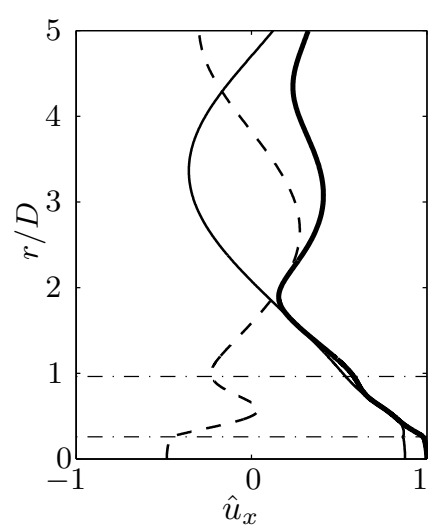

c) Shear-layer mode 1

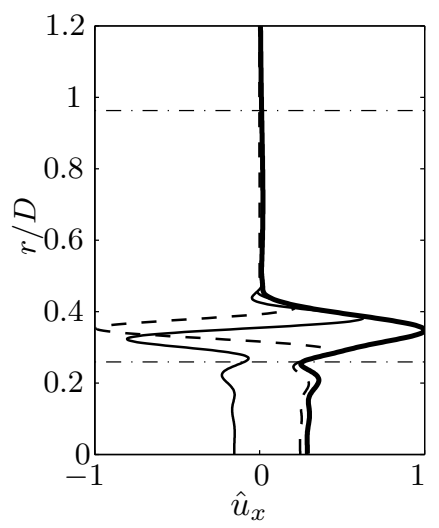

f) Core vorticity mode 1

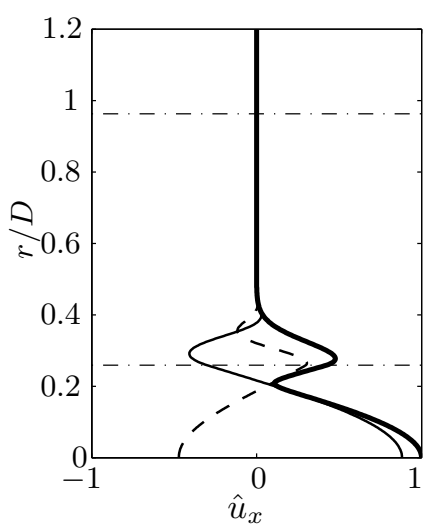

i) Acoustic mode 5

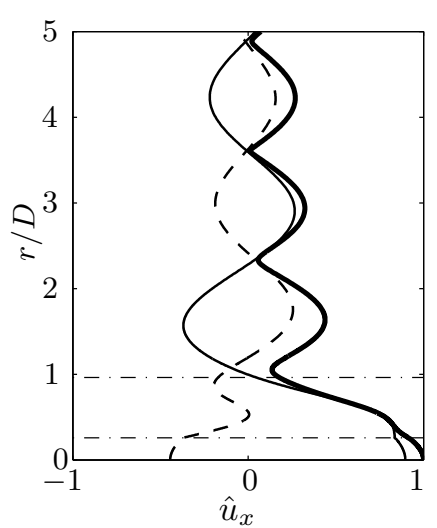

Figure 3. Mean velocity profile at $x=3 D(\mathbf{a})$, and (b-i) axial velocity component of some LST eigenfunctions for $S t=0.5$ and $m=0$. Thin solid and dashed lines correspond to real and imaginary parts, thick lines correspond to the absolute value. The dashed horizontal lines denote the radial extent of the mixing layer.

the projection is employed to extract, cross-section by cross-section, the contribution of the Kelvin-Helmholtz eigenmode to the total fluctuation field.

\section{A. Approximate projection using experimental data}

The determination of the weighting coefficients in the eigenmode decomposition (9) requires the evaluation of the inner product between the adjoint eigenfunction and the total fluctuation profile $\breve{\mathbf{q}}_{0}$ to be decomposed, 
Table 1. Sensitivity of the cofactor $C_{k}$ on the pressure and specific volume variables and on the domain truncation. $x=4 D$ and $m=0$.

\begin{tabular}{c|c|cc|cc|cc}
\hline & Exact & \multicolumn{2}{|c}{ Approx. } & \multicolumn{2}{c}{ Truncated } & \multicolumn{2}{c}{ Approx. and truncated } \\
$S t$ & $\left|C_{k}\right|$ & $\left|C_{k}\right|$ & $\varepsilon(\%)$ & $\left|C_{k}\right|$ & $\varepsilon(\%)$ & $\left|C_{k}\right|$ & $\varepsilon(\%)$ \\
\hline \hline 0.1 & 1.6817 & 1.7766 & 5.3 & 1.6299 & 3.2 & 1.7756 & 5.3 \\
0.2 & 1.6282 & 1.7231 & 5.5 & 1.5063 & 8.1 & 1.6825 & 3.2 \\
0.3 & 1.2346 & 1.1952 & 3.3 & 1.2650 & 2.4 & 1.2018 & 2.7 \\
0.4 & 1.2700 & 1.1662 & 8.9 & 1.3333 & 4.8 & 1.1950 & 6.3 \\
0.5 & 0.1692 & 0.1547 & 9.4 & 0.1740 & 2.8 & 0.1571 & 7.7 \\
0.6 & 0.1353 & 0.1278 & 5.8 & 0.1365 & 0.9 & 0.1284 & 5.3 \\
0.7 & 0.1477 & 0.1420 & 4.0 & 0.1481 & 0.3 & 0.1422 & 3.8 \\
0.8 & 0.0260 & 0.0255 & 1.8 & 0.0260 & 0.1 & 0.0255 & 1.8 \\
0.9 & 0.1950 & 0.1905 & 2.4 & 0.1951 & 0.1 & 0.1906 & 2.3 \\
\hline
\end{tabular}

that comprises all the five fluid variables in the problem $u_{x}, u_{r}, u_{\theta}, p$ and $\zeta$. When data obtained from numerical simulations are used, ${ }^{25}$ all the fluid variables are available and the projection can be done in an exact manner. When experimental data is used, only some of the variables are known: the present PIV provides the three velocity components but no information on the pressure or specific volume. Some works exist in the literature,$^{23}$ in which additional information, e.g. the absence of contribution from some families of eigenmodes, is exploited in order to close the problem as an algebraic system of equations for the weighting amplitudes. This kind of assumption is not possible for the present turbulent flow, as all the different families are expected to contribute to some extent. In this work, the contributions of the (unknown) pressure and specific volume fluctuations to the inner product are neglected. The relative importance of these variables is hypothetised to be small for the isothermal and low Mach number jet under consideration, as they account mostly to compressibility effects.

A second problem associated to the use of experimental data concerns the limited spatial resolution and domain that can be probed in the experiments. Due to the power limitation in the laser used, the timeresolved PIV setup used interrogates a relatively small spatial window on each plane; the data is truncated at a distance ranging between $0.66 \mathrm{D}$ and $0.82 \mathrm{D}$, depending on the axial location. While this extension is sufficient to contain the peak turbulent fluctuations, concentrated at the lipline, it may result to be too short for the accurate evaluation of (9).

In order to have a quantitative estimate of the error due to the approximations done, the cofactors $C_{k}=\left(\hat{\mathbf{q}}_{k}^{+}\right)^{*} \mathbf{L} \hat{\mathbf{q}}_{k}$ are computed for the Kelvin-Helmholtz eigenmode with and without neglecting the pressure and specific volume variables (the latter case referred to as approximated), and two truncations in the radial direction: $r=5 D$ corresponding to the domain boundary in the LST computations, and $r=0.66 D$, corresponding to the minimum extent of the PIV data. Table 1 shows the cofactors and the relative errors for different $S t$ for $m=0$, at $x=4 D$. Similar values are obtained for other axial locations. For the first helical mode the errors are found to be slightly lower. The errors in the cofactors introduced by the domain truncation and neglecting the pressure and specific volume contributions are expected to be of the same order as the errors introduced in the projection of experimental data. In view of table 1, the errors in the projected amplitudes are estimated to be less than $10 \%$ for the K-H eigenmode. Note that the adjoint eigenfunctions for different eigenmode families exhibit different radial decay rates, indicating different sensitivities to the data truncation.

\section{B. Projection on the Kelvin-Helmholtz eigenmode}

The contribution of the Kelvin-Helmholtz eigenmode from locally-parallel LST at each cross section where PIV measurements were performed is computed by means of the projection the experimental data on its adjoint eigenfunction. Along with the Fourier transform in the azimuthal direction, a finite-time Fourier transform is applied to the experimental data, resulting in a set of realizations, or Fourier-transform of data segments. This procedure is one standard step in the computation of power spectra, and the same cautions 
regarding the choice of frequency bin $\Delta S t$ and the highest reliable frequency apply.

The perturbation profiles corresponding to different segments may differ notably along the total time of the experiment having an strong impact on the projected amplitude. On the other hand, using the PIV profiles averaged over segments is not correct as the phase relationships are not adequately preserved in the averaging process. Instead, each Fourier-transformed segment is projected on the K-H eigenmode and the resulting amplitudes are then averaged, enabling direct comparisons between the measured velocity power spectral density and the extracted K-H eigenmode.

Figure 4 compares the jet axis velocity as measured using a traverse hot-wire, extracted from the PIV measurements, and its projection on the K-H eigenmode for $m=0$. The symmetries of the problem imply that only the axial velocity component of the $m=0$ Fourier mode contributes to the hot-wire measurements in a significant manner. ${ }^{16}$ The variance of the data projection is also shown, in order to illustrate the variability of the amplitudes over segments. As said in section II, 100 segments with a $50 \%$ overlap are used in the process. Some differences are observed between the hot-wire and PIV measurements. Due to the limitation in frequency imposed by the laser device, the PIV sampling frequency was only $S t=1.82$ resulting into higher spectral amplitudes using TR-PIV due to aliasing, as observed for the higher frequencies. A different kind of discrepancy is found up to $\approx 2 D$, where PIV presents higher amplitudes. This is attributed to different signal-to-noise ratios between PIV and hot-wire measurements. Hot wires are more appropriate for the measurement of small velocity fluctuations, such as the ones measured inside the potential core of the jet for low $x / D$. It was found that time-resolved PIV measured significantly higher amplitudes of velocity fluctuations inside the core; however, taking the first POD mode from the measurements filters the uncorrelated noise, leading to amplitudes which are close to the hot-wire results. ${ }^{18}$

The extracted K-H eigenmode is found to match well the evolution of the hot-wire measurements over the first diameters of streamwise evolution, both in absolute amplitude and spatial growth. It should be remarked that the K-H amplitudes are obtained from the PIV data and not from the hot-wire measurements, and thus are also affected by aliasing. The excellent agreement between the projected K-H eigenmode and hot-wire confirms that the discrepancies observed between PIV and hot-wire measurements are due to the mentioned uncorrelated noise in the PIV data at the axis. The adjoint K-H eigenfunctions peak at the lipline and decay very fast towards the jet axis, so physical or unphysical contamination at the axis have a very limited contribution to the projected amplitude, and are then filtered out.

The close match between the extracted K-H eigenmode and the hot-wire measurements extends for a distance of few diameters over the axial direction, that depends strongly on the frequency. The agreement is lost downstream and then the projection on the $\mathrm{K}-\mathrm{H}$ overestimates the centerline velocity. Note that the spatial growth of the extracted K-H eigenmode in figure 4 is not predicted by LST, but is imposed by the increase in the averaged projection amplitude that only considers the shape of the fluctuation profiles. Consequently, a good agreement in the projected amplitude implies a good agreement between the measured velocity profiles with the linear K-H eigenmode (as will be discussed in section VI), but not necessarily linear dynamics of the underlying physics. The overestimations past the first few diameters are attributed to strong differences between the measured fluctuations and the K-H eigenmode alone. If an accurate projection ${ }^{\mathrm{b}}$ of the experimental data were performed using a large number of eigenmodes, good agreement with the hot-wire velocity should be expected again.

The agreement between hot-wire measurements and the PIV data projection on the K-H eigenmode over a limited spatial region can be related to the variations of the LST eigenspectrum properties discussed in section IV. Region I is characterised by the well-defined potential core and a thin mixing layer, so the K-H eigenmode is unstable for all frequencies and clearly distinguished from other eigenmodes. It is found that at the axial locations where good agreement is found in figure 4, the $\mathrm{K}-\mathrm{H}$ eigenmode is always unstable. The agreement is gradually lost as the $\mathrm{K}-\mathrm{H}$ becomes stable and approaches and eventually merges with the branch of mixing-layer modes, as additional eigenmodes become relevant. This process happens in Region II as the potential core closes, and is strongly affected by the frequency. As we move further downstream, the branches of mixing-layer and core vortical eigenmodes coalesce. From this point, and all over Region III, there is no distinct K-H eigenmode anymore and projection results are not shown in figure 4 . Figure 5 repeats for clarity some of the results in figure 4, also showing the approximate boundaries of the different regions discussed above.

\footnotetext{
${ }^{\mathrm{b}} \mathrm{An}$ accurate bi-orthogonal projection comprising a large number of eigenmodes is not possible here due to the limitations of the experimental data used.
} 
a) $S t=0.1$
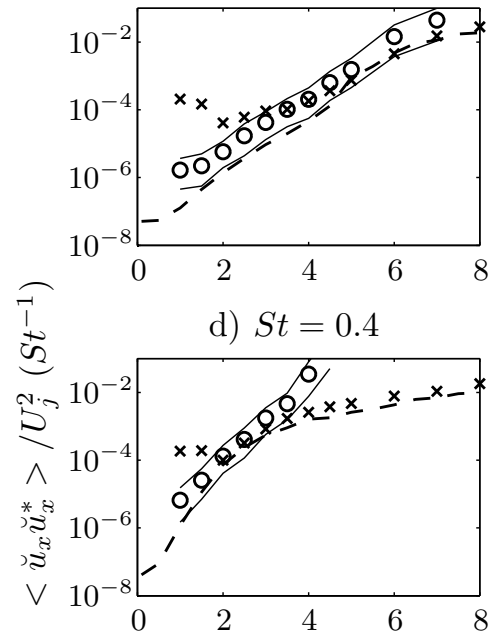

g) $S t=0.7$

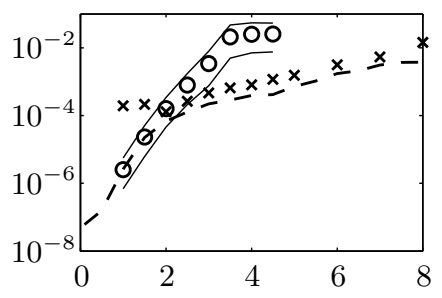

b) $S t=0.2$

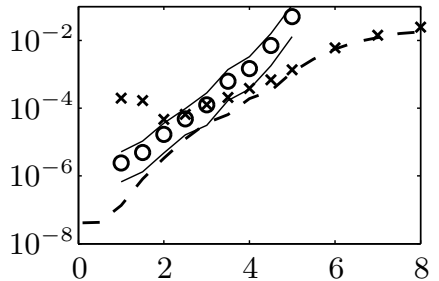

e) $S t=0.5$

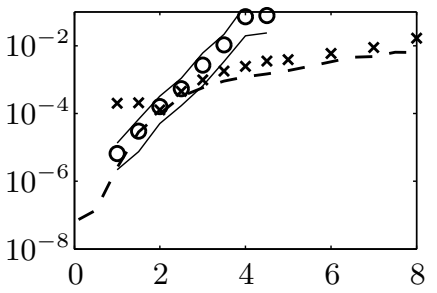

h) $S t=0.8$

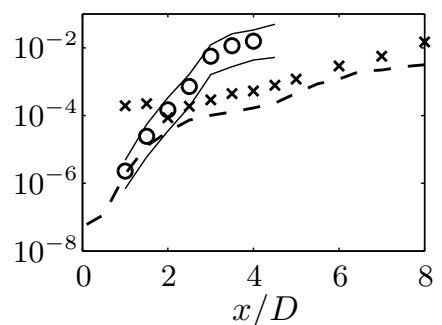

c) $S t=0.3$

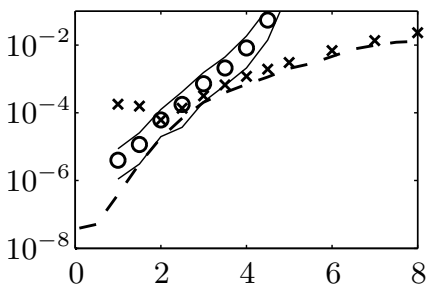

f) $S t=0.6$

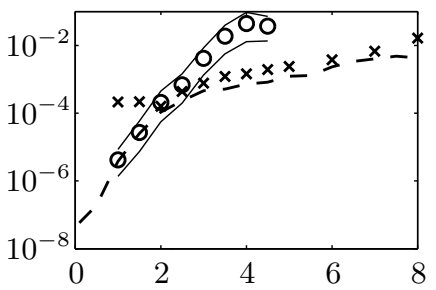

i) $S t=0.9$

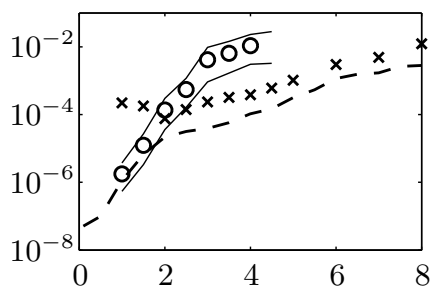

Figure 4. Power spectral density of the axial velocity fluctuation at the centerline: Hot-wire (dashed line); TR-PIV (crosses); projection on the K-H eigenmode (circles); projection on the K-H eigenmode \pm variance (thin solid line).

\section{Initial conditions for PSE wavepacket models}

In our previous research, ${ }^{18}$ linear PSE wavepackets were computed and compared with the hot-wire measurements. Linear models are arbitrary with respect to a constant amplitude factor $A_{\omega m}$ that must be determined using empirical data. The bi-orthogonal projection of experimental data is a theoretically-founded method of determining this amplitude. Linear stability theory for parallel flows shows that the Kelvin-Helmholtz eigenmode is the only source of linear amplification of the fluctuations in subsonic jets. Even if there is a broad fluctuation spectra at the nozzle lip due to the turbulent shear-layer, the Kelvin-Helmholtz eigenmode will grow and dominate the PSE solution at a short distance downstream, at least while it remains being unstable. Consequently, computing the projected amplitude of the experimental data on the K-H eigenmode at a single cross-section determines the PSE wavepacket amplitude completely. ${ }^{25,42}$

Not all cross-sections are adequate for the amplitude determination via adjoint projection. As seen in figure 4, only a region of few diameters from the nozzle delivers the correct amplitudes $a_{n}$ (equation 9) for the K-H eigenmode and can be used to determine $A_{\omega m}$ (equation 3 ). Figure 5 shows the centerline velocity corresponding to the PSE wavepackets scaled by the projection of the PIV data at a single cross-section. For all frequencies, the PSE computations are initiated at $x_{0}=0 D$ while the amplitude determination is done at a different cross-section due to the technical impossibility of measuring at the nozzle exit cross-section. The axial location where the amplitude is determined for each frequency can be identified in the figure, as the PSE solution and the extracted K-H eigenmode must be nearly coincident there. A small deviation is possible because the K-H eigenmode from LST and the PSE solution are not identical (comparisons are shown in section VI). 
a) $S t=0.1$
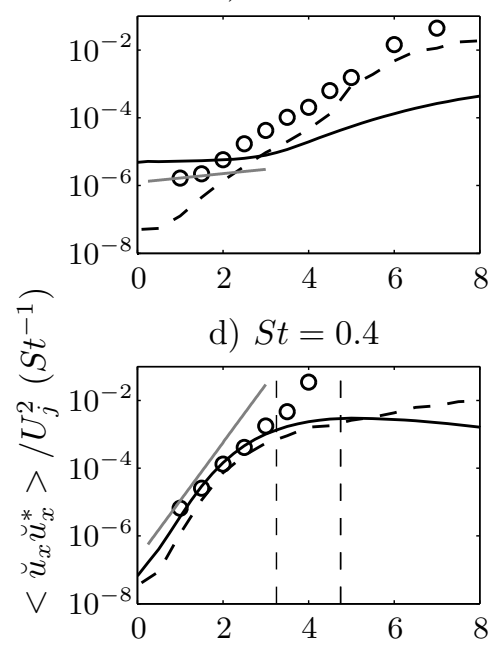

g) $S t=0.7$

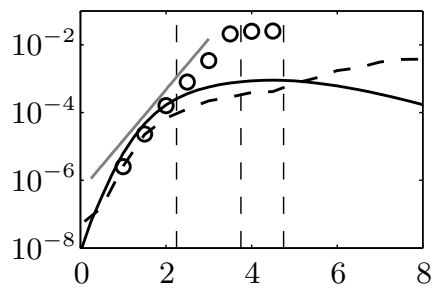

b) $S t=0.2$

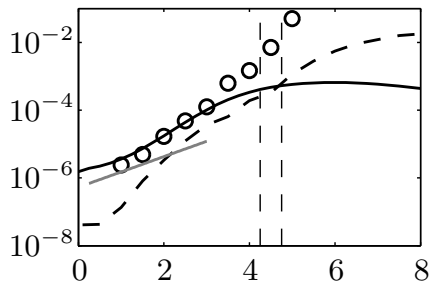

e) $S t=0.5$

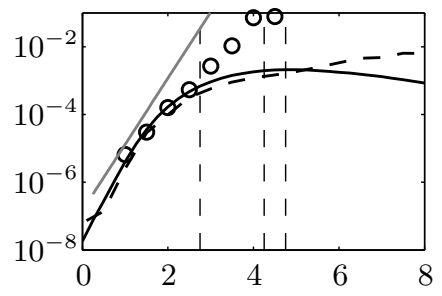

h) $S t=0.8$

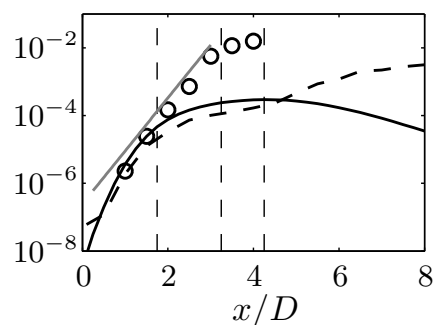

c) $S t=0.3$

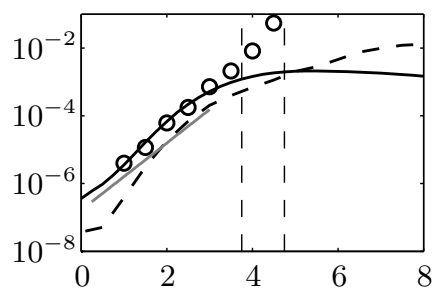

f) $S t=0.6$

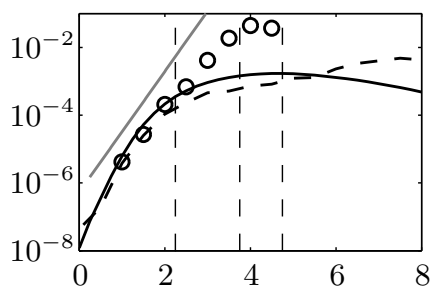

i) $S t=0.9$

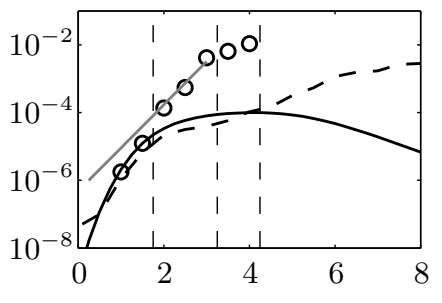

Figure 5. Power spectral density of the axial velocity fluctuation at the centerline: Hot-wire (dashed line); projection on the K-H eigenmode (circles); PSE solution (solid line). The vertical dashed lines correspond, respectively, to the axial location where K-H eigenmode becomes stable, where K-H eigenmode coalesces with the branch of shear-layer eigenmodes, and where the branches of shear-layer and core vorticity modes merge. The grey line shows the slope predicted by the local LST problem at $x=1.5 D$.

\section{Comparison of cross-stream velocity profiles}

This section compares the velocity profiles measured by stereoscopic PIV with those corresponding to the locally-parallel LST and PSE computations, discussed in the preceding sections. Again, PIV data is Fourier transformed in the azimuthal direction and time, allowing comparisons for each $(S t, m)$ pair.

In the past ${ }^{18}$ Proper Orthogonal Decomposition (POD) was used in order to extract the velocity fluctuations most coherent on each cross-section from the PIV measurements. Whereas the unfiltered experimental velocity profiles presented some discrepancies with the computed PSE velocity fields, the leading POD mode, containing most of the fluctuating kinetic energy, was shown to be in good agreement up to the potential core closure. POD modes are obtained by solving the integral equation

$$
\int R_{x, x}\left(x, r, r^{\prime}, S t, m\right) \xi_{x}\left(x, r^{\prime}, S t, m\right) r^{\prime} d r^{\prime}=\lambda(x, m, S t, m) \xi_{x}(x, r, S t, m),
$$

where $\xi$ is an eigenfunction (or POD mode), $\lambda$ is the corresponding eigenvalue and $R_{x, x}$ is given by

$$
R_{x, x}\left(x, r, r^{\prime}, S t, m\right)=u_{x}(x, r, S t, m) u_{x}^{*}\left(x, r^{\prime}, S t, m\right) .
$$

The kernel $R_{x, x}$ is not Hermitian; an auxiliar Hermitian kernel was obtained by multiplying the integral equation (10) by $\sqrt{r}$ and considering $R_{x, x}\left(x, r, r^{\prime}, S t, m,\right) \sqrt{r r^{\prime}}$ as the kernel and $\xi_{x}\left(x, r, r^{\prime}, S t, m\right) \sqrt{r^{\prime}}$ as the eigenfunction. In the following comparisons, only the first, most energetic POD mode for each $(S t, m)$ is considered:

$$
\operatorname{POD}\left(\breve{u}_{x}\right)=\sqrt{\lambda^{(1)}} \xi_{x}^{(1)} .
$$




\section{A. Axisymmetric mode at $S t=0.5$}

Comparisons of the axial and radial velocity profiles for $S t=0.5$ and $m=0$ at different axial sections are shown in figures 6 and 7. As known from previous work, the first POD and PSE solution agree closely in the first diameters from the nozzle lip, losing similarity gradually as we move downstream. In the first diameters, the K-H eigenmode from LST is similar to the PSE solution both in shape and amplitudes. This agreement starts deteriorating when the K-H eigenmode predicted by the LST problem becomes neutral and then stable, as discussed in section V. For $S t=0.5, m=0$ the K-H eigenmode becomes stable at $x \approx 3 D$. Afterwards, the velocity profiles corresponding to the $\mathrm{K}-\mathrm{H}$ eigenmode differs from the PSE solution. This discrepancy should be expected, as the PSE integration tends to follow the dominant (i.e. most unstable or less damped) eigenmode in the local LST eigenspectrum. When the $\mathrm{K}-\mathrm{H}$ eigenmode becomes stable at the beginning of the Region II, PSE no longer delivers an approximation of the discrete K-H eigenmode but a combination of different eigenmodes including part of the mixing-layer and core vorticity modes, that are nearly neutral or have a smaller decay rate. Consequently, PSE computations agree better with the PIV profiles that the $\mathrm{K}-\mathrm{H}$ eigenmode once the physical mechanism responsible of the wavepacket amplification is no longer active. As the contribution of many additional eigenmodes become relevant, the bi-orthogonal projection on the $\mathrm{K}-\mathrm{H}$ eigenmode no longer delivers meaningful predictions.

\section{B. First helical mode at $S t=0.5$}

Comparisons of the axial development of the velocity profiles for the mode $S t=0.5, m=1$ are done in figure 8. Again, good agreement is found between the K-H eigenmode and the PSE solution for the first two diameters, that is gradually lost as the $\mathrm{K}-\mathrm{H}$ eigenmode becomes stable at $x \approx 3 D$. The agreement is also very good with the first POD mode. Further downstream, PSE compares well with the POD mode until the end of the potential core both in shape and amplitude.

\section{Axisymmetric mode at $S t=0.2$}

Linear PSE wavepacket models have consistently been found to compare poorly with experiments and simulations of unforced turbulent jet flows at frequencies below $S t \approx 0.3 \cdot{ }^{15,16,18,42}$ The axial amplification of the PSE solution clearly underestimates the measured evolution, as shown in figures 4 and 5 . At least two possible explanations were proposed ${ }^{15}$ to this mismatch. One possibility is that the separation of scales required by the PSE approach is not verified by the lower frequencies, as the characteristic wavelengths become comparable to the length of the potential core. The second possibility is that the neglected nonlinear interactions, that seem to have very limited effect at the higher frequencies, are important at the lower frequency range. The present results suggest that nonlinearity is the cause of the disagreement, as discussed next.

Figure 5 shows that projection of the experimental fluctuations on the $\mathrm{K}-\mathrm{H}$ eigenmode overestimates the amplitude of the hot-wire measurements by a constant factor for every cross-section, an error that can be attributed to aliasing in the PIV measurements. However, the downstream amplification of the projected K$\mathrm{H}$ eigenmode follows closely the one observed in the hot-wire measurements, up to $x \approx 4.5 D$. The agreement in the slopes is more evident for $S t=0.1$. Note that the evolution of the amplitude of the projected K-H eigenmode is not governed by the growth rate predicted by LST, but only by the changes in the experimental data. The amplification predicted by LST at $x=1.5 \mathrm{D}$ is shown in figure 5 for comparison, and is similar to the amplification predicted by linear PSE.

Comparisons of the axial velocity profile for $S t=0.2$ and $m=0$ at different axial sections are shown in figure 9. The shape of the PSE and K-H eigenmode profiles agree well for most of the cross-sections, while differences in the amplitude appear as we move downstream. Comparisons with the first POD mode are not as good as for $S t=0.5$, but the main features of the profile, such as the lipline peak and the phase jump about $r=0.7 D$ agree well. The agreement in the velocity profiles predicted by linear models (LST and PSE) with the experimental data suggests that the mean flow divergence is not responsible for the underestimation of the spatial amplification. On the other hand, weakly nonlinear models usually employed in the study of laminar-turbulent transition share some of the characteristics observed here. In the context of transitional flow, weakly nonlinear growth stands for the amplification of subharmonic modes by nonlinear forcing due to another frequency mode, the evolution of which remains approximately linear. ${ }^{49}$ These models rely on the so-called shape assumption, ${ }^{50}$ that refers to the observation that the fluctuation profiles remain almost identical to those of the linear LST in conditions of finite amplitudes and relevant nonlinear excitation. 
a) $x=1 D$

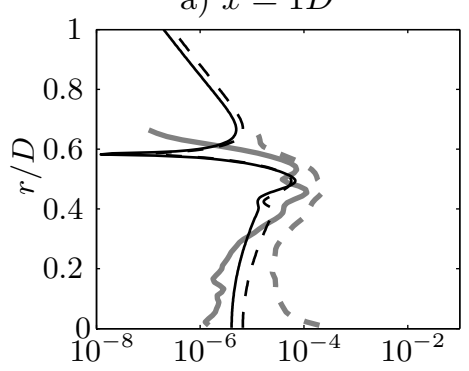

d) $x=2.5 D$

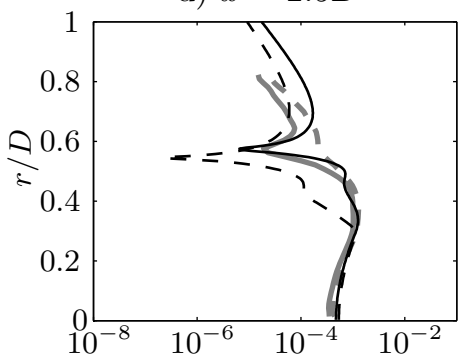

g) $x=4 D$

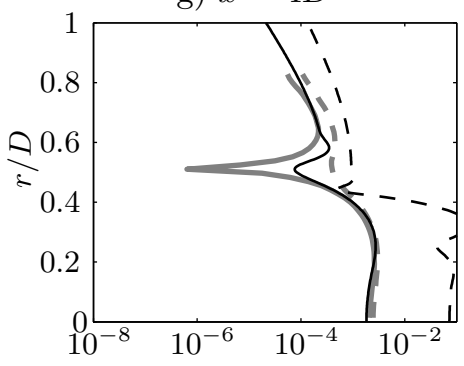

b) $x=1.5 D$

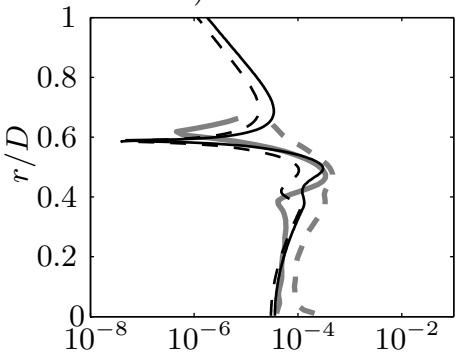

e) $x=3 D$

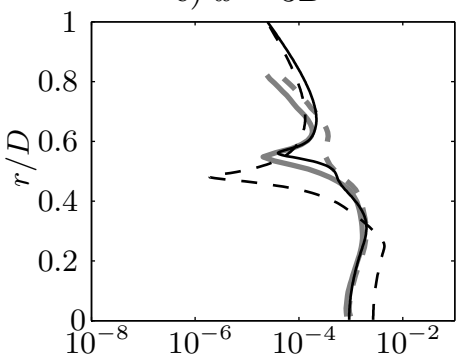

h) $x=4.5 D$

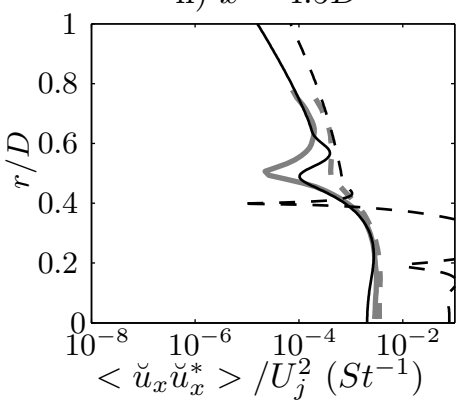

c) $x=2 D$

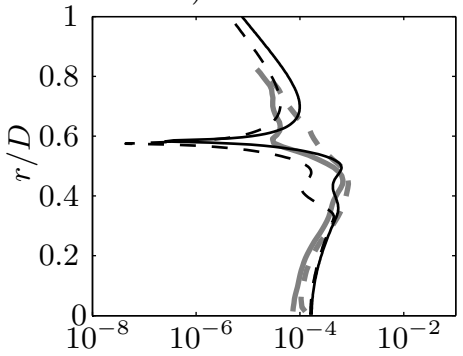

f) $x=3.5 \mathrm{D}$

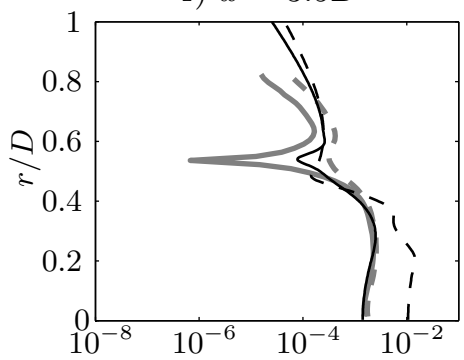

i) $x=5 D$

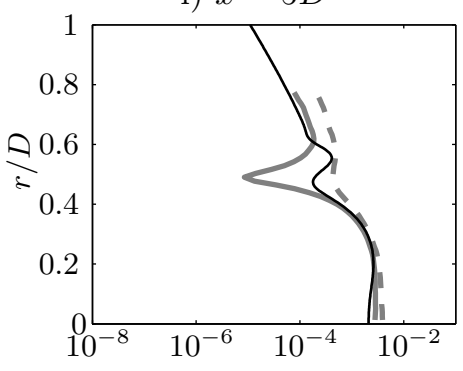

Figure 6. Comparison between the profiles of axial velocity component $\breve{u}_{x}$ at different cross-sections for Fourier mode $S t=0.5, m=0$ : Raw PIV data (dashed grey); POD-filtered PIV data (solid grey); projection on K-H eigenmode (dashed black); PSE solution (solid black).

Weakly nonlinear models have been shown to reproduce experimental observations in boundary layers, ${ }^{51,52}$ and also the initial stages of nonlinear generation of subharmonics describing the vortex formation in plane mixing-layers. In the case of unforced natural turbulence, a broad range of frequencies can contribute to the nonlinear amplification of the lower frequencies due to the inverse turbulent cascade.

\section{Conclusions}

Our previous work ${ }^{18}$ on the identification and modeling of the velocity field associated with the wavepackets existing in subsonic turbulent jet flow showed very good agreement between the velocity field measured using time-resolved PIV and the instability waves computed as linear PSE solutions, up to the end of the potential core. This observation are in line with the spatial amplification rates measured using hot-wire velocimetry at the jet axis. However, when proceeding downstream the agreement is lost both for the amplitudes and shape functions.

The present work further investigates this question. A detailed study is done of the properties of the parallel-flow LST eigenspectrum and their evolution along the streamwise direction. Subsequently, PIV measurements at cross-sections are used into a bi-orthogonal projection in order to determine the contribution of the Kelvin-Helmholtz eigenmode to the total fluctuation field, as well as to determine the amplitudes of the computed PSE solutions. Comparisons between the fluctuation profiles corresponding to experimental 
a) $x=1 D$

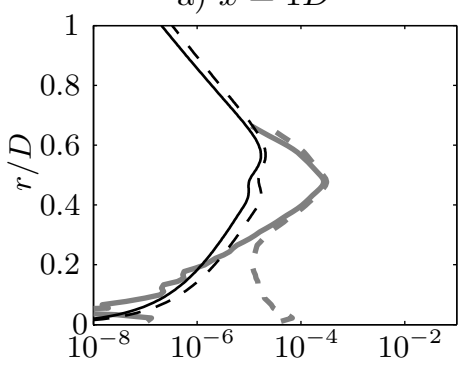

d) $x=2.5 \mathrm{D}$

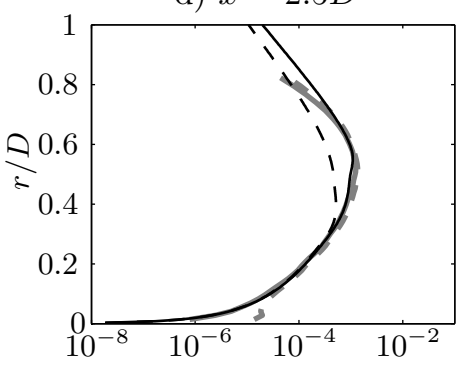

g) $x=4 D$

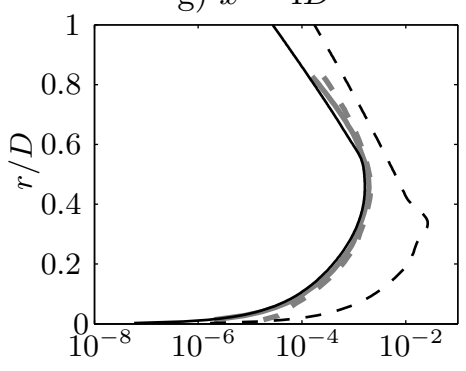

b) $x=1.5 \mathrm{D}$

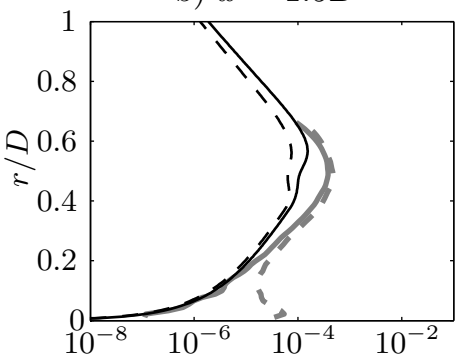

e) $x=3 D$

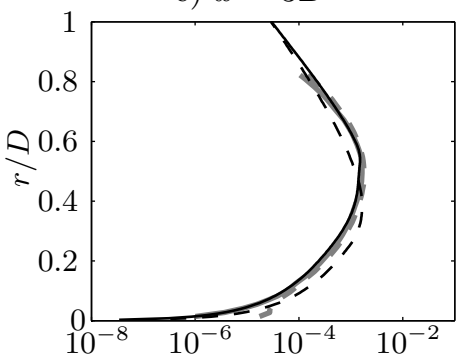

h) $x=4.5 \mathrm{D}$

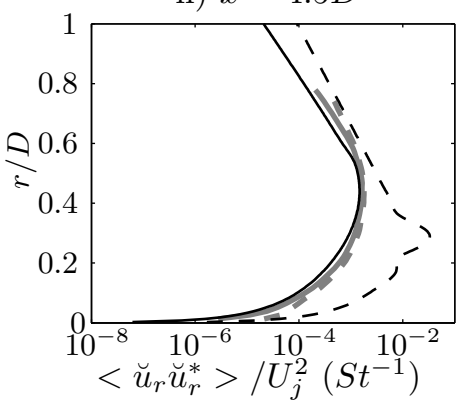

c) $x=2 D$

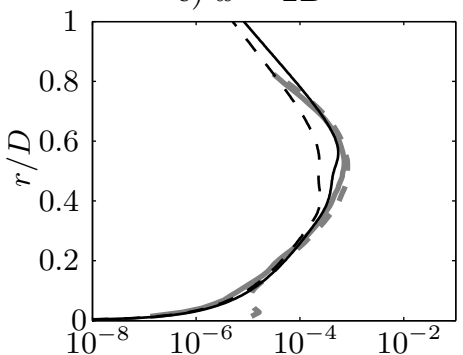

f) $x=3.5 \mathrm{D}$

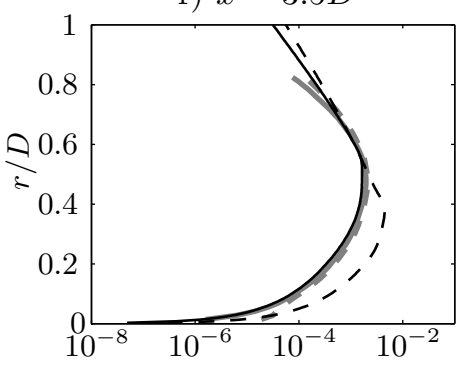

i) $x=5 \mathrm{D}$

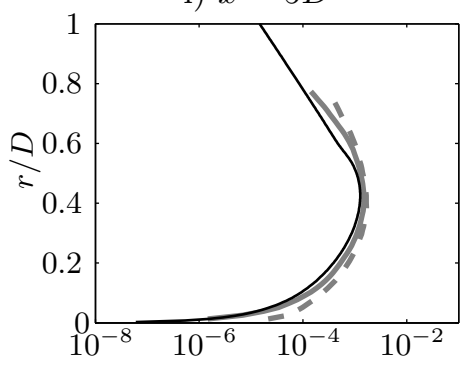

Figure 7. Comparison between the profiles of radial velocity component $\breve{u}_{r}$ at different cross-sections for Fourier mode $S t=0.5, m=0$ : Raw PIV data (dashed grey); POD-filtered PIV data (solid grey); projection on K-H eigenmode (dashed black); PSE solution (solid black).

data with the projected K-H eigenmode and the PSE solution, and their evolution along the streamwise direction, can be explained in terms of changes in the LST eigenspectrum. Three different spatial regions are found, as follows:

Region I is related to the initial mixing layer and extends up to the transition region where the mixinglayer thickness becomes comparable to the radial extent of the potential core. In this region the K-H eigenmodes unstable and the wavepackets suffer a marked amplification. Good agreement is found between the PSE and K-H velocity profiles in the first diameter from the nozzle, but they differ from the experimental ones. Incidentally, the amplitude of the projection on the K-H eigenmode agrees well with experiment, and imposing this amplitude to the PSE solution results in good agreement with the experimental wavepackets downstream. These observations suggest that the first diameter from the nozzle is characterised by a transition from the fluctuations internal to the nozzle to the inflectional instability observed downstream. As the fluctuations unrelated to the wavepacket decay, good agreement is observed between the K-H eigenmode, the PSE solution and the first POD mode.

A Region II appears next, in which the PSE solution and the experimental profile agree, but the K-H eigenfunction does not. A single K-H eigenmode is still identified, but it is stable and eventually merges with a branch of fluctuations localised in the relatively thick mixing layer. As the Kelvin-Helmholtz eigenmodes is no longer the dominant eigenmode, i.e. the mechanism responsible for the wavepacket amplification cease, the PSE solution is not longer governed by the K-H eigenmode and captures a combination of other eigenmodes that decay slower. This observation explains the differences between models based on the K-H eigenmode 
a) $x=1 D$

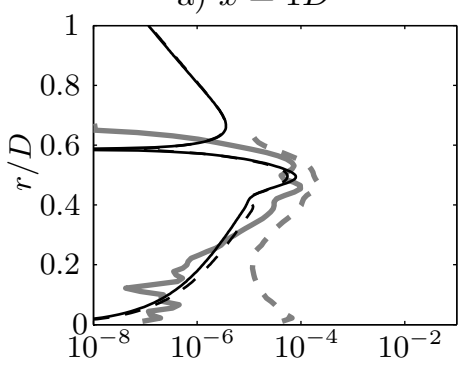

d) $x=2.5 D$

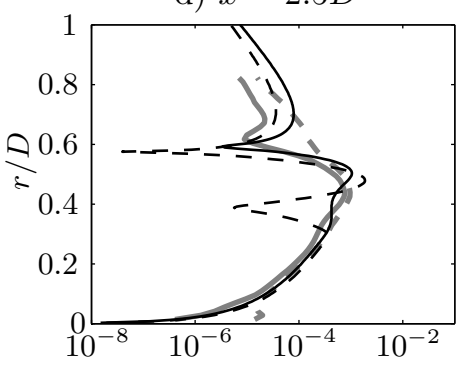

g) $x=4 D$

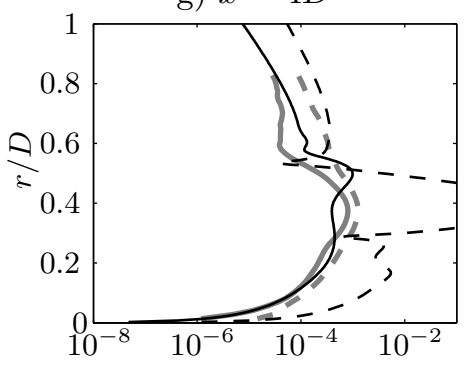

b) $x=1.5 D$

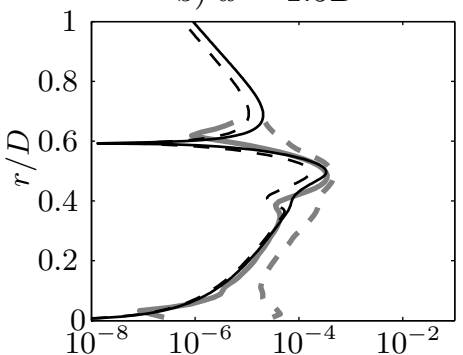

e) $x=3 D$

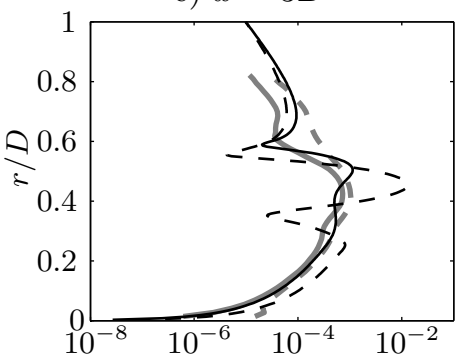

h) $x=4.5 D$

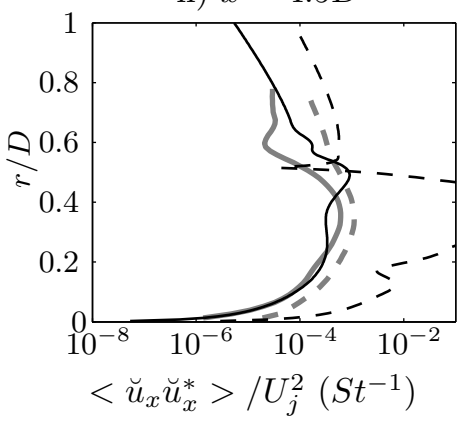

c) $x=2 D$

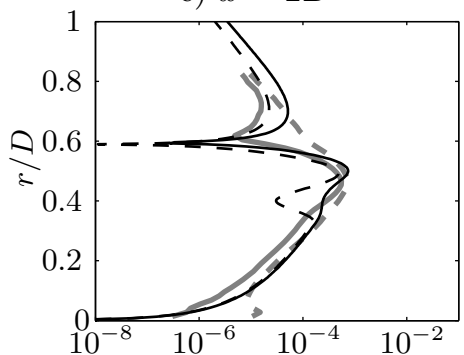

f) $x=3.5 D$

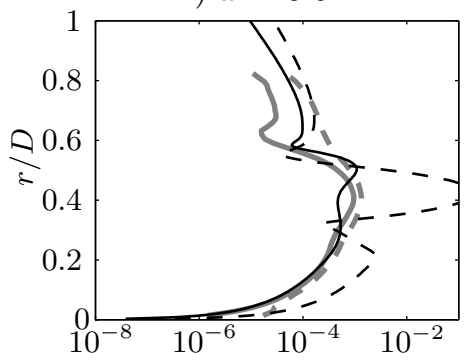

i) $x=5 D$

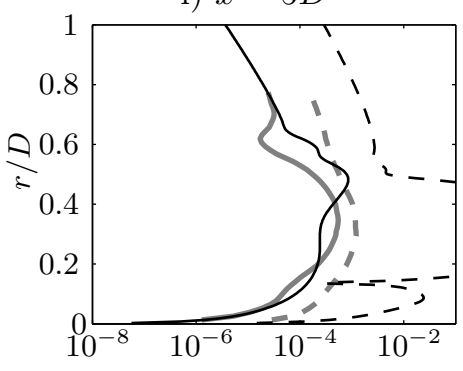

Figure 8. Comparison between the profiles of axial velocity component $\breve{u}_{x}$ at different cross-sections for Fourier mode $S t=0.5, m=1$ : Raw PIV data (dashed grey); POD-filtered PIV data (solid grey); projection on K-H eigenmode (dashed black); PSE solution (solid black).

alone $\mathrm{e}^{3,6,32,53}$ ) and PSE computations past the amplitude peak (Regions II and III), while they are nearly coincident in Region I. In this region, the wavepackets constitute the dominant velocity fluctuations in the experiment and POD is an efficient method for their eduction.

Agreement between the linear PSE models and the cross-sectional velocity profiles is finally lost at the end of the potential core, coinciding with the commence of the Region III in the evolution of the LST eigenspectrum. With the disappearance of the potential core, the branches of mixing-layer and core eigenmodes coalesce. Again, the PSE solution in this region is an admixture of different eigenmodes, but is still representative of the downstream linear evolution of the wavepacket. Whether the discrepancies found between the experimental measurements and PSE models is due to nonlinear interactions past the potential core is not clarified by the present results. In related works, ${ }^{12,15}$ near-field pressure measurements have been compared to the same linear PSE solutions discussed here, showing good agreement downstream of the potential core. These results suggest that the wavepackets persist in the region of developed turbulence but account for relatively low kinetic energy levels, making their identification in the velocity field difficult.

The projection of the measured fluctuations on the dominant instability eigenmode presented in this work enabled us to study two additional aspects of the PSE wavepacket models. Linear PSE computations have been shown to deliver good comparisons with wavepacket signatures at frequencies $S t \geq 0.3$, while underpredicting the growth at lower frequencies. The good agreement found between the amplification of centerline velocity in hot-wire measurements and the projected $\mathrm{K}-\mathrm{H}$ eigenmode over some diameters suggests that nonlinear excitation may be significant at these low frequencies, as suggested by Suponitsky et al. ${ }^{54}$ 
a) $x=1 D$

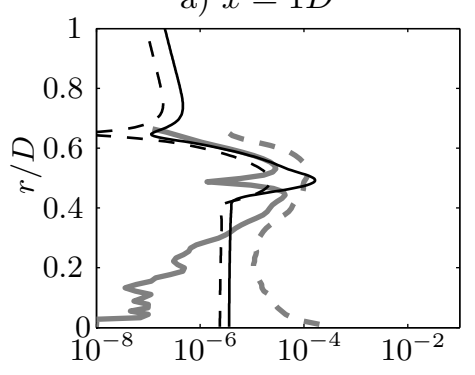

d) $x=2.5 \mathrm{D}$

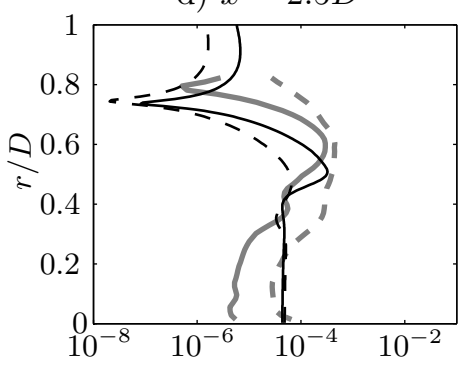

g) $x=4 D$

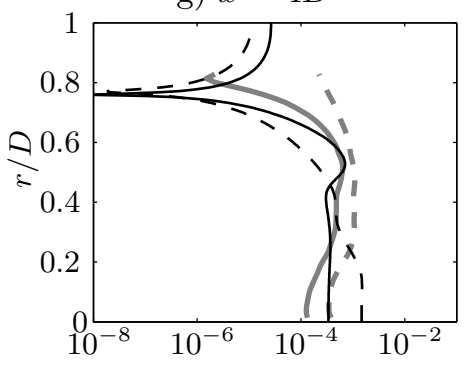

b) $x=1.5 \mathrm{D}$

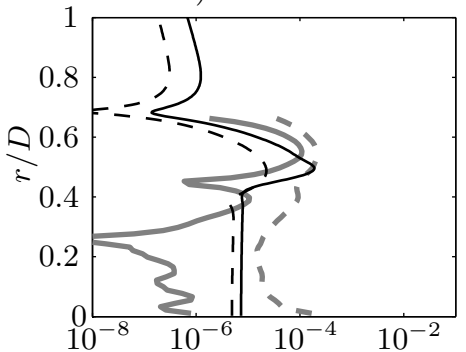

e) $x=3 D$

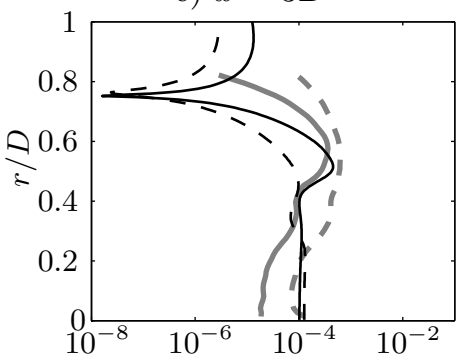

h) $x=4.5 \mathrm{D}$

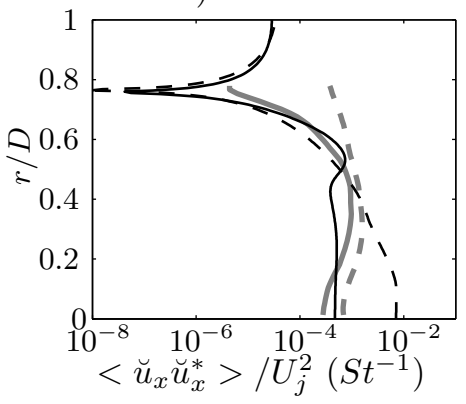

c) $x=2 D$

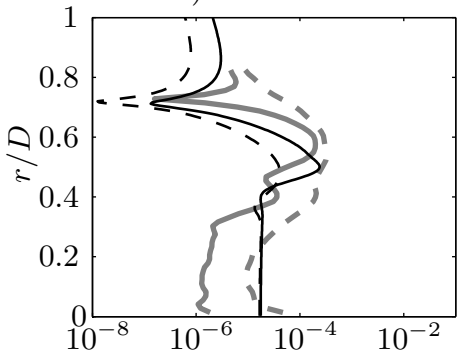

f) $x=3.5 \mathrm{D}$

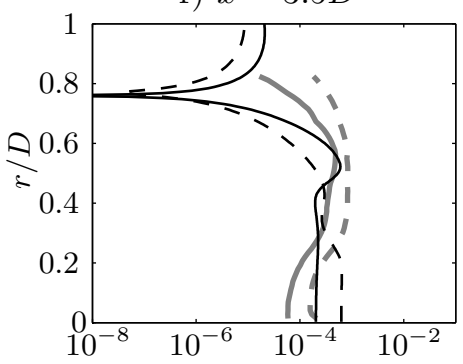

i) $x=5 \mathrm{D}$

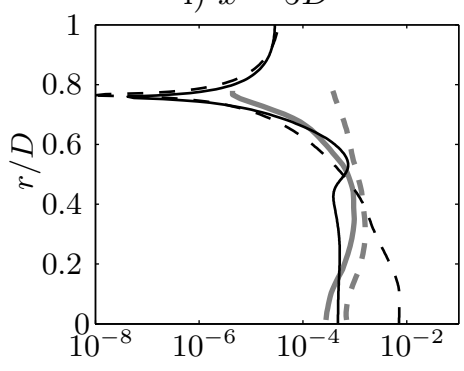

Figure 9. Comparison between the profiles of axial velocity component $\breve{u}_{x}$ at different cross-sections for Fourier mode $S t=0.2, m=0$ : Raw PIV data (dashed grey); POD-filtered PIV data (solid grey); projection on K-H eigenmode (dashed black); PSE solution (solid black).

Finally, the bi-orthogonal projection of empirical data on the Kelvin-Helmholtz is proposed as valid method fto determine the initial amplitude in linear PSE models. If sufficient information on the fluctuations at a near-nozzle cross-section is available, the projection determines the amplitude of the wavepacket without further knowledge of the downstream evolution of the fluctuations.

\section{Acknowledgments}

We thank Drs. Kristjan Gudmundsson, Arnab Samanta and Aniruddha Sinha for their contributions on the development of the PSE code and Carine Fourment-Cazenave, Patrick Braud and Dr. Joël Delville for their work during the experiments. The work of D. Rodríguez was supported by the EU Marie Curie COFUND program.

\section{References}

${ }^{1}$ G. A. Brès, J. W. Nichols, S. K. Lele, F. E. Ham, Towards best practices for jet noise predictions with unstructured large eddy simulations, AIAA Paper 2012-2965.

${ }^{2}$ S. C. Crow, F. H. Champagne, Orderly structure in jet turbulence, J. Fluid Mech. 3 (1971) 547-591.

${ }^{3}$ C. Tam, D. Burton, Sound generated by instability waves of supersonic flows. Part 2. Axisymmetric jets., J. Fluid Mech. 
138 (1984) 273-295.

${ }^{4}$ E. Mollo-Christensen, Jet noise and shear flow instability seen from an experimenter's viewpoint, J. Applied Mech. 34 (1967) $1-7$.

${ }^{5}$ A. Michalke, Instabilititat eines Kompressiblen Runden Freistrahls unter Berucksichtigung des Einflusses der Strahlgrenzschichtdicke, Zeitschrift für Flugwissenschaften 19 (1971) 319-328.

${ }^{6}$ C. Tam, P. Morris, Tone excited jets - Part V: A theoretical model and comparison with experiments, J. Sound Vib. 102 (1985) 119-151.

${ }^{7}$ J. T. C. Liu, Developing large-scale wavelike eddies and the near jet noise field, J. Fluid Mech. 62 (1974) $437-464$.

${ }^{8}$ R. Mankbadi, J. T. C. Liu, A study of the interactions between large-scale coherent structures and fine-grained turbulence in a round jet, Proc. Roy. Soc. London 1443 (1981) 541-602.

${ }^{9}$ P. Morris, M. Giridharan, G. Lilley, On the turbulent mixing of compressible free shear layers, Proc. R. Soc. Lond. A. 431 (1990) 219-243.

${ }^{10}$ T. Suzuki, T. Colonius, Instability waves in a subsonic round jet detected using a near-field phased microphone array, J. Fluid Mech. 565 (2006) 197-226.

${ }^{11}$ C. E. Tinney, P. Jordan, The near pressure field of co-axial subsonic jets, Journal of Fluid Mechanics 611 (2008) $175-204$.

${ }^{12}$ D. E. S. Breakey, P. Jordan, A. V. G. Cavalieri, O. Léon, Near-field wavepackets and the far-field sound of a subsonic jet, 19th AIAA/CEAS Aeroacoustics Conference and Exhibit, 27-29 May, AIAA, Berlin, Germany, 2013.

${ }^{13}$ F. P. Bertolotti, T. Herbert, P. Spalart, Linear and nonlinear stability of the blasius boundary layer, J. Fluid Mech. 242 (1992) 441-474.

${ }^{14}$ T. Herbert, Parabolized stability equations, Annu. Rev. Fuid Mech. 29 (1997) 245-283.

${ }^{15}$ K. Gudmundsson, T. Colonius, Instability wave models for the near-field fluctuations of turbulent jets, J. Fluid Mech. 689 (2011) 97-128.

${ }^{16}$ D. Rodríguez, A. Samanta, A. Cavalieri, T. Colonius, P. Jordan, Parabolized Stability Equation models for predicting large-scale mixing noise of turbulent round jets, AIAA Paper 2011-2838.

${ }^{17}$ D. Rodríguez, A. Sinha, G. A. Brès, T. Colonius, Parabolized Stability Equation models in turbulent supersonic jets, AIAA Paper 2012-2117.

${ }^{18}$ A. Cavalieri, D. Rodríguez, P. Jordan, T. Colonius, Y. Gervais, Wavepackets in the velocity field of turbulent jets, submitted to J. Fluid Mech.

${ }^{19}$ A. Michalke, H. V. Fuchs, On turbulence and noise of an axisymmetric shear flow, J. Fluid Mech. 70 (1975) $179-205$.

${ }^{20}$ J. Laufer, T. C. Yen, Noise generation by a low-Mach-number jet, J. Fluid Mech. 134 (1983) 1-31.

${ }^{21}$ A. Tumin, M. Amitay, J. Cohen, M. Zhou, A normal multimode decomposition method for stability experiments, Phys. Fluids 8 (10) (1996) 2777-2779.

${ }^{22}$ A. Tumin, Multimode decomposition of spatially growing perturbations in a two-dimensional boundary layer, Phys. Fluids 15 (2003) 2525-2540.

${ }^{23}$ A. Tumin, Three-dimensional spatial normal modes in compressible boundary layers, J. Fluid Mech. 586 (2007) 295-322.

${ }^{24}$ A. Tumin, X. Wang, X. Zhong, Direct numerical simulation and the theory of receptivity in a hypersonic boundary layer, Phys. Fluids 19 (11) (2007) 014101.

${ }^{25}$ D. Rodríguez, A. Sinha, G. A. Brès, T. Colonius, Inlet conditions for wave packet models in turbulent jets based on eigenmode decomposition of les data, Physics of Fluids under revision.

${ }^{26}$ A. Cavalieri, P. Jordan, T. Colonius, Y. Gervais, Axisymmetric superdirectivity in subsonic jets, J. Fluid Mech. 704 (2012) 388-420.

${ }^{27}$ T. Troutt, D. McLaughlin, Experiments on the flow and acoustic properties of a moderate reynolds number supersonic jet, J. Fluid Mech. 116 (1982) 123-156.

${ }^{28}$ P. Balakumar, Prediction of supersonic jet noise, AIAA Paper 1998-1057.

${ }^{29}$ M. R. Malik, C. L. Chang, Nonparallel and nonlinear stability of supersonic jet flow, Computers and Fluids 29 (2000) $327-365$.

${ }^{30}$ E. Piot, G. Casalis, F. Muller, C. Bailly, Investigation of the pse approach for subsonic and supersonic hot jets. detailed comparisons with les and linearized euler equations results, Int. J. Aeroacoustics 5 (2006) $361-393$.

${ }^{31}$ P. Ray, L. Cheung, S. Lele, On the growth and propagation of linear instability waves in compressible turbulent jets, Physics of Fluids 21 (2009) 054106.

${ }^{32}$ D. G. Crighton, M. Gaster, Stability of slowly diverging jet flow, J. Fluid Mech. 77 (2) (1976) $397-413$.

${ }^{33}$ K. Thompson, Time dependent boundary conditions for hyperbolic systems, J. Comp. Phys. 68 (1987) 1-24.

${ }^{34}$ K. Mohseni, T. Colonius, Numerical treatment of polar coordinate singularities, J. Comp. Phys. 157 (2000) $787-795$.

${ }^{35}$ F. Li, M. R. Malik, Spectral analysis of parabolized stability equations, Comp. and Fluids 26 (3) (1997) $279-297$.

${ }^{36}$ C. Grosch, H. Salwen, The continuous spectrum of the Orr-Sommerfeld equation. Part I. The spectrum and the eigenfunctions, J. Fluid Mech. 87 (1978) 33-54.

${ }^{37}$ H. Salwen, C. Grosch, The continuous spectrum of the Orr-Sommerfeld equation. Part II. Eigenfunction expansions, J. Fluid Mech. 104 (1981) 445-465.

${ }^{38}$ A. M. Tumin, A. V. Fedorov, Spatial growth of disturbances in a compressible boundary layer, J. Appl. Mech. Tech. Phys. 24 (1983) 548-554.

${ }^{39}$ D. Hill, Adjoint systems and their role in the receptivity problem for boundary layers, J. Fluid Mech. 292 (1995) $183-204$.

${ }^{40}$ A. M. Tumin, A. V. Fedorov, Excitation of instability waves in a boundary layer on a vibrating surface, J. Appl. Mech. Tech. Phys. 24 (1983) 670-674.

${ }^{41}$ A. Fedorov, A. Tumin, Initial-value problem for hypersonic boundary-layer flows, AIAA J. 41 (3) (2003) $379-389$.

${ }^{42}$ D. Rodríguez, A. Sinha, G. A. Brès, T. Colonius, Acoustic field associated with parabolized stability equations models in turbulent jets, 19th AIAA/CEAS Aeroacoustics Conference and Exhibit, 27-29 May, AIAA, Berlin, Germany, 2013. 
${ }^{43}$ A. E. Gill, Instabilities of Top-Hat jets and wakes in compressible fluids, Physics of Fluids 8 (1965) 1428-1430.

${ }^{44}$ C. Tam, F. Hu, On the three families of instability waves of high-speed jets, J. Fluid Mech. 201 (1989) $447-483$.

${ }^{45} \mathrm{P}$. J. Morris, The spatial viscous instability of axisymmetric jets, J. Fluid Mech. 77.

${ }^{46} \mathrm{~F}$. Bertolotti, T. Colonius, On the noise generated by convected structures in a Mach 0.9 hot, turbulent jet, AIAA Paper 2003-1062.

${ }^{47}$ H. Schlichting, Boundary layer theory, 7th Edition, McGraw-Hill, 1979.

${ }^{48}$ A. Michalke, Survey on jet instability theory, Prog. Aero. Sci. 21 (1984) 159-199.

${ }^{49}$ A. D. D. Craik, Nonlinear resonant instability in boundary layers, J. of Fluid Mech. 50 (1971) 393-413.

${ }^{50}$ T. Herbert, Secondary instability of boundary layers, Annu. Rev. Fuid Mech. 20 (1988) 487-526.

${ }^{51}$ Y. S. Kachanov, V. V. Kozlov, V. Y. Levchenko, Nonlinear development of a wave in a boundary layer, Proc. USSR Acad. Sci, Fluid Mech. 3 (1977) 49-35.

${ }^{52}$ Y. S. Kachanov, Physical mechanisms of boundary layer transition, Annu. Rev. Fluid Mech. 26 (1) (1994) $411-482$.

${ }^{53}$ P. Plaschko, Helical instabilities of slowly diverging flows, J. Fluid Mech. 92, part 2 (1979) 209-215.

${ }^{54}$ V. Suponitsky, N. Sandham, C. Morfey, Linear and nonlinear mechanisms of sound radiation by instability waves in subsonic jets, Submitted to J. Fluid Mech. 658 (2010) 509-538. 